\title{
Normal-Tissue Tolerance to Radiopharmaceutical Therapies, the Knowns and the Unknowns
}

\author{
Richard L. Wahl ${ }^{1}$, George Sgouros ${ }^{2}$, Amir Iravani ${ }^{1}$, Heather Jacene ${ }^{3}$, Daniel Pryma ${ }^{4}$, Babak Saboury ${ }^{5}$, Jacek Capala ${ }^{5}$, and \\ Stephen A. Graves ${ }^{6}$ \\ ${ }^{1}$ Mallinckrodt Institute of Radiology, Washington University, St. Louis, Missouri; ${ }^{2}$ Department of Radiology, Johns Hopkins University, \\ Baltimore, Maryland; ${ }^{3}$ Dana-Farber Cancer Institute, Boston, Massachusetts; ${ }^{4}$ Penn Medicine, University of Pennsylvania, \\ Philadelphia, Pennsylvania; ${ }^{5}$ National Institutes of Health, Bethesda, Maryland; and ${ }^{6}$ University of Iowa, Iowa City, Iowa
}

\section{$\mathbf{R}$} as they improve survival in patients with common diseases such as metastatic prostate cancer $(1,2)$. However, whereas sodium iodide $\left({ }^{131} \mathrm{I}\right)$ therapy has been used for over $70 \mathrm{y}$ in treating malignant and benign thyroid diseases, we are still in a learning phase in relation to understanding the toxicity from radiopharmaceutical therapies (RPTs). Much of what is considered "known" regarding radiopharmaceutical dose-toxicity relationships is the result of questionable extrapolation from $100+$ years of experience with external-beam radiation therapy (EBRT). Although this may have been a reasonable starting point, there are critical differences between EBRT and RPTs.

With external-beam irradiation, dose-response relationships have been informed by a set of measurements of the predicted and actual absorbed dose delivered to normal tissues and tumors. There have been great advancements in external-beam radiation dose delivery, including intensity modulated radiation therapy, as well as treatments in which tumor doses are intensified in specific areas informed by imaging, so called biologically guided radiation therapy $(3,4)$. These advancements have increased control and accuracy in dosing, leading to better patient outcomes.

Several things are clear regarding EBRT. First, external-beam radiation dosimetry to tissues is well-developed and there have been major efforts to standardize methods for absorbed dose measurement among radiation therapy centers globally. It is thus expected that absorbed dose estimates are likely within $<10 \%$ of one another among sites performing external-beam irradiation (5). In addition, whereas there can be "dose painting" to specific areas of tumor, it is typically the case that the absorbed dose from external beam is quite uniformly delivered in a given volume of tissue treated in a specific part of the body. It has also been clear since early studies with EBRT that radiation delivery to part of an organ is less likely to cause toxicity than radiation therapy of the entire organ. Early reporting of external-beam radiation toxicity in, for example, the liver or kidneys was influenced by the percentage of the organs irradiated, with partial organ irradiation less toxic than whole organ irradiation (i.e., one third of the liver could be irradiated to a higher dose without toxicity than irradiation of the entire

Received Aug. 24, 2021; revision accepted Oct. 15, 2021.

For correspondence or reprints, contact Richard L. Wahl (rwahl@wustl.edu). COPYRIGHT @ 2021 by the Society of Nuclear Medicine and Molecular Imaging. DOI: 10.2967/jnumed.121.262751 liver, and a part of the kidney could be irradiated to a higher level than a whole [or both] kidneys without systemic toxicity) (6).

Recently, a new approach using personalized treatment planning accounting for the biologic effect of a given radiation dose has been considered $(7,8)$. This approach calls for characterizing radiation dose not only in physical terms of energy deposition (absorbed dose, Gy) but also in terms of biologic effects on the tumor and normal tissue $(9,10)$. All limitations of the knowledge relevant to external radiation therapy are also of concern in RPT.

However, despite the long experience with EBRTs, there remain unanswered questions regarding the effects of absorbed dose rate (standard fractionation vs. hypofractionation), the use of radiotherapy in pediatric patients versus adults, optimal delivery of brachytherapy, optimal use cases for proton and particle therapy, patient-specific biologic factors increasing or decreasing the risk of toxicity, and potential interactions of external-beam radiation with other cancer therapies that may affect the response to radiation.

Compared with external-beam radiation, assessing radiopharmaceutical toxicity is in its relative infancy, or at most early adolescence. The biologic effect of a radiopharmaceutical agent is fundamentally based on "radiation effect" and "energy deposition in tissue," similar to external-beam radiation effect. However, there are at least 4 fundamental differences in normal-tissue response to radiation from RPT versus external-beam radiation: 1. Spatial nonuniformity of energy deposition by RPT (spatial domain); 2. Absorbed dose rate (temporal domain); 3. Importance of tissue microenvironment and microscale dosimetry (scale domain); and 4 . Time variation and paramount importance of pharmacokinetics (systems domain).

There are some additional considerations for RPT versus EBRT, including the knowledge that RPT is generally a systemic therapy and therefore partial-organ irradiation is not typically performed. Additionally, low-energy $\beta$-particle, Auger electrons, or $\alpha$-particles are associated with considerable nonuniformity of absorbed dose. The nonuniformity in absorbed dose is also tied to the spatial distribution of the RPT. Nonuniformity in the spatial distribution may arise due to expression of the molecular target in normal tissues (e.g., prostate specific membrane antigen [PSMA] expression in the salivary glands) or due to physiologic processing/transport of the agent (e.g., retention of most low molecular weight agents in the kidneys). Also related to these nonuniformities is the fact that current 3-dimensional imaging modalities - SPECT and PEThave the resolution and counting statistics needed to quantify macroscale nonuniformities (e.g., kidney renal cortex vs. overall kidney 
volume) but not assess nonuniformities at the microscale (e.g., renal tubule vs. renal glomerulus) level. The latter require modelbased activity apportionment and microscale $\mathrm{S}$ values $(11,12)$. Thus, continued early studies examining normal organ dose response are likely to depend strongly on the scale at which the agent localizes nonuniformly and on its emission properties.

Elsewhere in this supplement (13), early results are provided from the Society of Nuclear Medicine and Molecular Imaging (SNMMI) Dosimetry Task Force "challenge," showing that even when analyzing the same imaging data, varying laboratories can have different estimates of radiation-absorbed dose to specific tissues of relevance. These differences likely result from nonunified approaches to curve fitting and volume of interest definition among other factors. Indeed, other variables such as dose calibrator performance/calibration, camera sensitivity and calibration, as well as how attenuation, scatter, resolution, and partial-volume factors are addressed, can cause significant variability in estimates of radiation-absorbed dose to tumor and normal tissues. In general, these effects are more impactful on smaller and deeper-situated structures such as tumors, as the variability in dose estimates appears to be less in larger organs (14). That said, planar estimates of lung absorbed dose are, in the authors' opinion, highly variable depending on the selection of the precise background region of interest. Assessments of dose/response/toxicity are only as good as the estimation of activity concentration in particular organ volume (15). Similarly, estimates of radiation-absorbed dose to the bone marrow can be more challenging if the bone marrow dosimetry is estimated from planar imaging as opposed to SPECT.

Another consideration in linking radiation-absorbed dose in normal tissues to organ toxicity is whether the dosimetry estimates are obtained from the diagnostic companion to the therapeutic agent (as in the case of some theranostic pairs) (16) or from imaging the biodistribution of the therapeutic radiopharmaceutical itself, such as in the case of posttreatment imaging. In principle, the 2 should be highly correlated with a well-selected theranostic pair, but it is likely that a more "true" absorbed dose estimation may be obtained from posttreatment imaging (“dose validation"). Imaging posttherapy activity distributions can have problems as well, such as dead time issues in some $\gamma$-cameras with therapeutics with high photon flux, such as ${ }^{131} \mathrm{I}$, which may degrade absorbed dose rate estimates from early-timepoint data, unless major corrections are implemented. Similarly, septal penetration with high-energy $\gamma$-emitters such as ${ }^{131} \mathrm{I}$ can degrade quantitation. With pure $\beta$-emitters and $\alpha$-emitters, imageable photon flux via Bremsstrahlung radiation is limited and difficult to use for dosimetry. In these cases, paired theranostic imaging may yield equivalent or improved organ-specific dose estimates in practice.

Variability in radiation-absorbed dose to normal tissues can occur among patients receiving the same number of radioactive molecules in their therapy. Larger patients will have the radiopharmaceutical diluted into a larger volume, although many larger molecules such as radiolabeled antibodies (and many other radiopharmaceuticals) do not substantially accumulate in fat. Thus, dosing some radiopharmaceuticals based on body weight, or lean body mass, can be an imperfect normalizing process. In addition, there can be variable clearance rates of molecules from tissue to tissue and patient to patient. Thus, consideration must be given to recognize that there are population-based absorbed doses to organs, and there are patient-specific absorbed doses, which can substantially diverge from the population average. Notably, some molecules such as radioantibodies, which cross react with normal tissues, can have considerable variability in their clearance from patient to patient, and depending on the mass of molecules injected, possibly due to cross-reactivity with normal tissues. Patients receiving murine monoclonal antibodies may have rapid clearance of the radioantibody if there are human antimouse antibodies present or if a low protein mass is given versus an unlabeled antibody predose before the radioactivity. Similarly, patients lacking a spleen may have much slower clearance of radioantibody from the blood, and thus higher organ doses/administered activity than in patients with an intact spleen, this having been seen with anti-CD20 antibodies (16). Thus, one must distinguish between individual dosimetry and average population dosimetry in absorbed dose/response/toxicity estimates. As dosimetry methods are harmonized and clinically implemented, we anticipate greater availability of patient-specific dosimetry data that can be linked to organ toxicity.

These admitted uncertainties in radiopharmaceutical dosimetry estimates lead to some variability in the dose-response relationships that have been demonstrated for RPTs. As an example, estimates of absorbed dose to the salivary glands from ${ }^{131} \mathrm{I}$ therapies are being improved by the availability of PET imaging with ${ }^{124} \mathrm{I}$ as compared with planar imaging. Until recently, it has been difficult to perform treatment escalation studies based on organ-absorbed dose because of the relatively cumbersome process of evaluating dosimetry with a suitable theranostic pair. These studies need to be done in greater numbers and are a major opportunity to better refine our understanding of normal-tissue absorbed-dose response.

\section{MECHANISM OF IRRADIATION FROM RPT}

Radiation can damage both normal tissues and tumors in several ways. Classically, radiation-induced damage to tissues occurs when ionizing radiation, either as a direct event or through the generation of oxygen- free radical, damages DNA. Although single-stranded DNA breaks can be repaired effectively, breaks in both strands of DNA can result in irreversible damage. A variety of events can occur due to this including deletions of segments of DNA or repair of DNA with less than perfect fidelity. Such a loss of DNA integrity can lead to failed cell proliferation and cell death. The cell membrane and mitochondria can also be damaged by radiation, and such damage can induce apoptosis $(17,18)$.

The multiple molecular mechanisms for radiation-induced cell death are complex and beyond the scope of this review. In short, they include downstream effects of mitotic catastrophe and mitotic death, apoptosis, necrosis, senescence, autophagy, and possibly other pathways including necroptosis and ferroptosis. It is also increasingly appreciated that not just cell intrinsic factors are involved, but also the microenvironment, including immunogenic cell death enhanced by radiation (19).

Virtually all tissues are made of a variety of components. Blood vessels supply nearly every tissue of relevance. Thus, in some cases, the radiation tolerance of a specific tissue may be related to the radiation tolerance of a component of a tissue. For example, in the brain the astrocytes and glial cells proliferate slowly and may themselves be less sensitive to radiation than the blood vessels or supporting cells for the vessels.

In general, tissues that proliferate very slowly are less radiosensitive and will demonstrate radiation induced damage much later than more rapidly proliferating tissues $(20)$. The rapidly proliferating cell populations within bone marrow, skin, testes, and gut are particularly radiosensitive, but can recover very quickly from radiation. These are sometimes called acute responding tissues. The brain, kidneys, and bone are less immediately sensitive, but are 
slow to recover (if ever) from radiation-induced damage. The liver is also slow to respond to radiation damage, but it can recover reasonably quickly. Although it is generally the case that slowly proliferating tissues are not radiosensitive, an exception are small lymphocytes, which proliferate slowly but quickly undergo apoptosis after exposure to relatively low doses of radiation.

Although $\beta$ - and $\gamma$-radiation are viewed as having a relative biologic efficiency (RBE) of 1.0, other emerging RPTs, particularly those that use radionuclides that emit $\alpha$-particles, carry much a higher RBE (21). $\alpha$-particles, which are helium nuclei with an atomic mass of 4 , carry much more energy per disintegration and deposit it at a relatively short distance and thus are considered a form of high-LET (linear energy transfer) radiation. Consequently, a single $\alpha$-particle traversal of a cell nucleus can cause multiple double-strand breaks and likely lead to cell death (22). Although DNA damage is not the only way tissues are injured by radiation, it is one of the classical events described in radiobiology literature as a major cause of cell death. It is commonly acknowledged that $\alpha$-particles have an RBE value 3-7 times higher that of $\beta$-particles. Although most forms of radiation exert DNA effects primarily through intermediary oxygen free radicals, $\alpha$-particles are more likely to interact directly with the DNA (23).

\section{DOSE RATE, BIOLOGICALLY EFFECTIVE DOSE (BED), AND MICRODISTRIBUTION OF DOSE}

In general, the higher the absorbed dose rate, the more substantial the normal tissue (and tumor) toxicity is per dose unit ( $\mathrm{J} / \mathrm{kg}, \mathrm{Gy}$ ). This is in part because high dose rate radiation exposures do not allow normal tissue or tumor to repair substantially. Conventional standard-fraction external-beam radiotherapy is typically given in $1.8-2.0 \mathrm{~Gy} / \mathrm{d}$ fractions over 6 5-d weeks $(\sim 10 \mathrm{~Gy} / \mathrm{wk})$. The VISION trial delivered ${ }^{177} \mathrm{Lu}$ in sequential treatments, depositing $\beta$-radiation dose to tumors over a total period of approximately 6 mo (1). Although the dose rate with RPT is usually thought of as lower than that of external-beam radiation, there are some situations, such as with short-lived radioisotopes, where a single administered activity can deliver relatively high time-averaged absorbed doses over a relatively short period of time. For example, ${ }^{90} \mathrm{Y}$ microspheres can deliver a high absorbed dose in a relatively short period of time (e.g., $10 \mathrm{~d}$ ) from a single treatment. An additional confounding factor when considering absorbed dose is the issue of DNA repair kinetics relative to the rate of radiation-induced damage. Two therapies with equivalent time-averaged dose delivery may result in differing biologic effects due to differences in instantaneous dose rate (i.e., Gy/min from EBRT vs. cGy/min from RPT).

For therapies using radionuclides emitting particles with longer range, such as high-energy $\beta$-emitters or emitters with a significant fraction of $\gamma$-radiation, microscale dosimetry may be less relevant. However, with shorter-pathlength $\beta$-emitters such as ${ }^{177} \mathrm{Lu}$ or with $\alpha$ - or Auger-particle emitters, the particles travel only a relatively short distance, so their microscale dosimetry in tissue is of much greater importance. As an example, ${ }^{90} \mathrm{Y}$ as a more energetic $\beta$-particle emitter when attached to octreotate causes renal toxicity in humans, whereas ${ }^{177} \mathrm{Lu}$-DOTATATE is much less renal-toxic, likely due to the differential microscale dosimetry in the kidneys, as ${ }^{177} \mathrm{Lu}$ is a lower energy $\beta$-emitter (24).

\section{NORMAL-TISSUE DOSE LIMITS}

A considerable effort has been undertaken to understand the relationships of EBRT dose and normal-tissue toxicity. A seminal paper by Emami et al. (6) summarized normal-tissue tolerances to external photon irradiation as they were known in 1991, and these data have been gradually updated and expanded through other initiatives such as Quantitative Analysis of Normal Tissue Effects in the Clinic (QUANTEC) and Hypofractionated Treatment Effects in the Clinic (HyTEC) $(25,26)$. These resources present normaltissue dose limits in terms of TD5/5 and TD50/5, the total doses associated with a complication rate of $5 \%$ and $50 \%$ within 5 y, respectively, as introduced by Rubin and Cassarett in 1972 (27). Dose limits recommended within these works were the product of high-quality published data, expert opinion, and model-based extrapolation when deviating from typical dose and fractionation schedules (i.e., $2 \mathrm{~Gy}$ per treatment fraction). These documents have helped to shape current radiation therapy clinical practice and as such they may act as a "road map" for establishing appropriate normal-tissue dose limits for RPTs.

Normal-tissue dose limits are a function of absorbed dose rate (or dose fractionation schedule in the case of EBRT), radiation quality ( $\alpha$ vs. $\beta^{-}$; microdistribution), tissue type, and time between treatments. These concepts are detailed in other articles within this journal supplement $(28,29)$. Despite biologic sensitivity to various factors, it is often practical to present dose limits or dose effects in terms of absorbed dose for a particular radiopharmaceutical and treatment pattern, while acknowledging that accurate comparison between different radiopharmaceuticals or radiation modalities requires careful modeling of radiobiologic effects. As such, in the sections below we discuss normal-tissue toxicity from radiopharmaceuticals in terms of absorbed dose from individual therapies. A summary of normal tissues and associated dose limits is provided in Table 1, including tissues for which dose limits from RPT are not currently known. Normal-tissue dose limits are partly known for a handful of organs in the body for specific radiopharmaceuticals; however, it is clear that less is known regarding RPT normal-tissue dose limits in comparison to EBRT treatment techniques. Careful evaluation of the intermodality differences among organs that have been characterized for both RPT and EBRT may inform methods for extrapolation to RPT limits in organ tissues without published data. It is increasingly clear from clinical experiences with RPT that the external-beam-derived organ dose limits do not consistently predict toxicity from radiopharmaceuticals and so these limits should not be strictly enforced and, instead, radiopharmaceutical-specific dose limits are required. Dose escalation studies driven by modern dosimetry are also important to consider, pursuant to avoiding systematic underdosing of patients.

\section{Bone Marrow}

The ability to image and assess the biodistribution of the radiopharmaceutical in patients, before treatment, or after the first fraction of a fractionated treatment regimen, make it possible to identify potential dose-limiting tissues. That salivary gland or renal toxicity may be of concern for PSMA-targeting small molecules but not for PSMA-targeting antibodies is apparent to a nuclear medicine physician by visual inspection of images corresponding to each agent at an appropriately chosen time after administration. Ideally, such imaging information, along with properties of the radionuclide, would be used to estimate the absorbed dose and, at minimum, distinguish between a range of administered activities that will be safe versus a range that will lead to toxicity. Although, there are many confounding factors (the unknowns), this process is at the heart of what gives RPT an advantage over treatment modalities that do not incorporate imaging and dosimetry. 


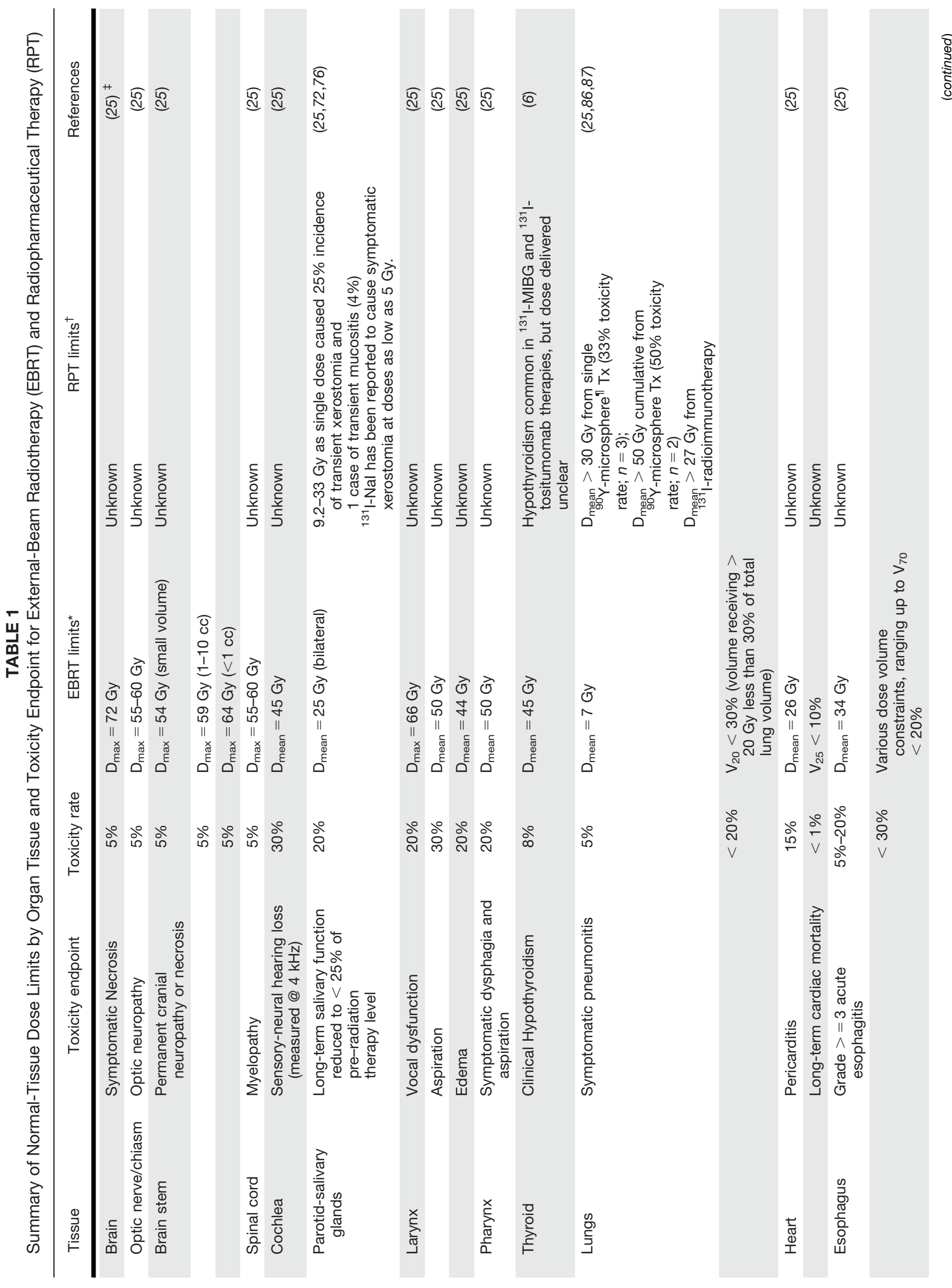




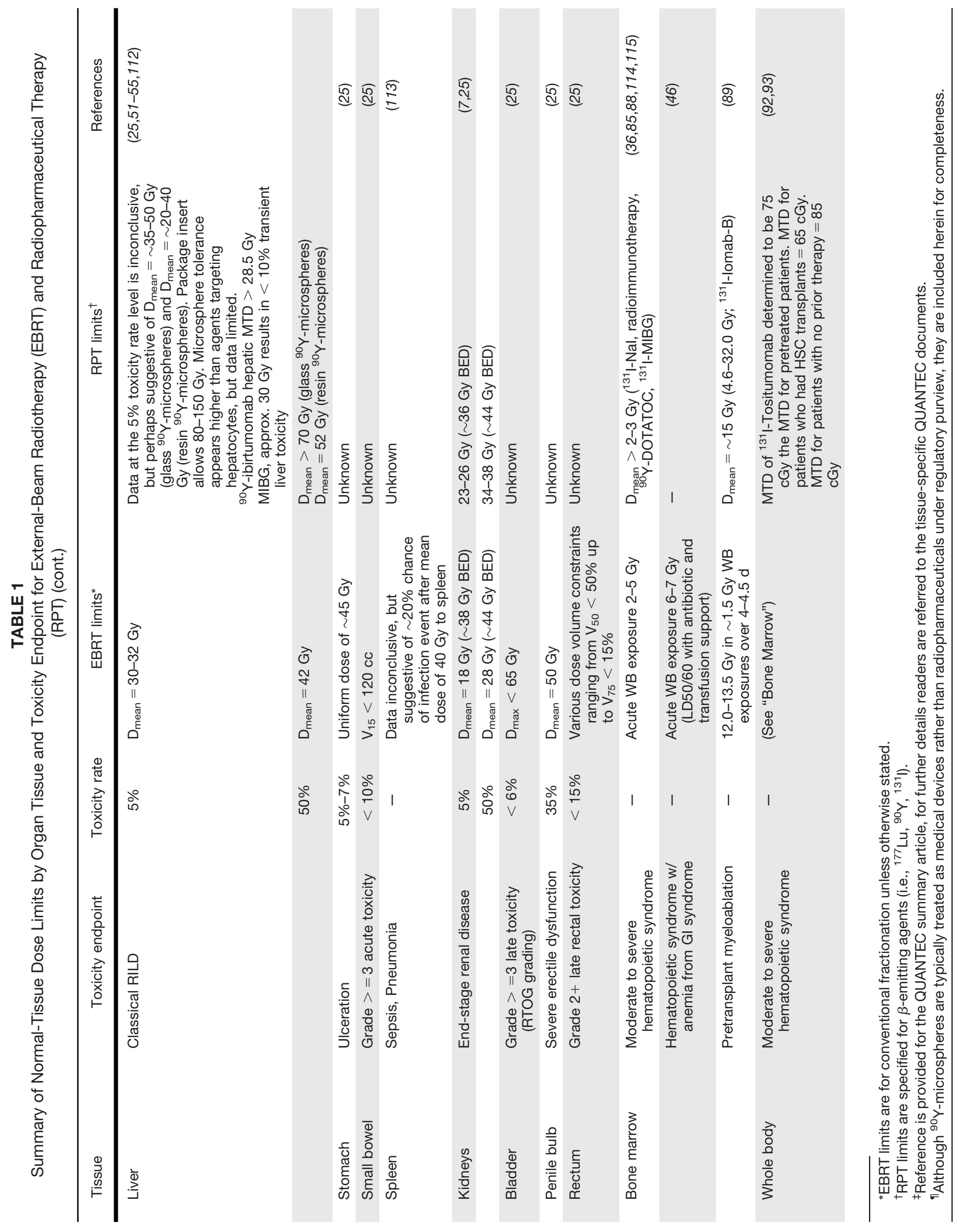


RPT agents are usually administered systemically (intrathecal, intracavitary, and hepatic artery injections are some of the exceptions); the potential for hematologic toxicity will therefore depend on a combination of inherent marrow radiosensitivity, prior patient exposure to hematotoxic agents, and the absorbed dose to the marrow. A thorough, but now dated, review of marrow dosimetry focused on radiolabeled antibodies was published in 2000 (30). Best practice guidelines for assessing hematologic toxicity in RPT have also been published (31).

Because the marrow is a distributed organ, direct image-based quantification of the time-integrated activity (TIA) (28) in the marrow is certainly possible; it would require delineation of all marrow-containing regions to identify all of the marrowcontaining voxels on PET/CT or SPECT/CT imaging. These could be used to calculate the TIA and from this absorbed dose to the entire marrow. In practice, this is not done-rather, "marrow-rich, low background" regions (e.g., lumbar vertebrae L3 to L5) are segmented and used to extract a TIA concentration that is then used to estimate red marrow absorbed dose (32-34). Although a blood-based approach has been described (35) and used to show that red marrow absorbed dose better predicts hematologic toxicity for antibody-based RPT (36), the imaging-based approach is preferred because it does not assume a red marrow-to-blood activity concentration ratio that is constant over time $(33,37)$ and that applies only to antibodies (38). More significantly, image-based red marrow dosimetry may also be applied when the RPT binds to marrow, bone, or blood components or in situations where there is the possibility of cancer cell infiltration of the marrow. This scenario is common for many RPTs agents, and perhaps most prominently for prostate cancer $(39,40)$, where metastatic dissemination is coincident with the marrow. In fact, the paper by Violet et al. illustrates a technique by which dosimetry for a distributed tissue - tumor metastases in this case - is achieved (39).

Compartmental modeling may also be used to estimate red marrow absorbed dose (41-43). Whole-body absorbed dose has also been used as a surrogate for marrow toxicity (44). For ${ }^{131} \mathrm{I}$-antiCD20 antibodies, a whole-body absorbed dose of 75 cGy was established as the maximum-tolerated dose (MTD) in patients with non-Hodgkin lymphoma who had been heavily pretreated with chemotherapy (16).

Red marrow dosimetry for $\alpha$-particle emitter RPT $(\alpha$ RPT) requires consideration of the microscale distribution of the TIA. This is because the short, $50-80 \mu \mathrm{m}$ range of $\alpha$-particles can lead to a highly nonuniform dose distribution to the degree that the average absorbed dose over the marrow volume may not predict biologic effects. Average marrow absorbed dose from antibody, peptide, or small molecule-based $\alpha$ RPT is likely to predict hematologic toxicity whereas bone targeting agents such as ${ }^{223} \mathrm{RaCl}_{2}$ will overestimate the potential biologic impact of a calculated average marrow absorbed dose (45). Red marrow dosimetry for $\alpha$ RPT agents also must account for the biodistribution of free daughters (46). The special considerations associated with $\alpha$ RPT dosimetry have been previously reviewed $(21,47)$.

With marrow absorbed dose estimates, it is relevant to consider that the marrow is quickly damaged by radiation but can, within limits, regenerate and be able to be treated again. This approach has been taken with chemotherapy for many years, with multiple cycles and intervening times without treatment for the marrow to reconstitute. As an example, ${ }^{131}$ I-tositumomab therapy was successfully repeated at a $75 \mathrm{cGy}$ total body dose level at months to years after initial treatment, with no evidence of additive toxicity (48). This differs from what we expect to be the case for slower regenerating tissues such as the liver or kidneys, where we view absorbed dose levels as cumulative and additive toward an upper limit.

Although 2-3 Gy is considered the maximum-tolerated radiation-absorbed dose to the marrow, based on perhaps limited dosimetric data, these limits are those present when the marrow is expected to reconstitute on its own. When stem cell transplant is considered and there is or is not tumor involvement in the marrow, the dosimetric limit is much higher, likely reflecting the tolerance of stem cells to re-engraft in the marrow, which has been ablated by radiation. In studies using ${ }^{131} \mathrm{I}$-anti-CD45 antibodies, a possible marrow dose of up to $48 \mathrm{~Gy}$ has been considered acceptable. Early analyses of the clinical data from 49 patients who received ${ }^{131} \mathrm{I}-$ apamistimab show the absorbed dose delivered to marrow (median, 14.7 Gy; range, 4.6-32 Gy) allowed for marrow re-engraftment, thus suggesting the MTD to marrow (with reconstitution) exceeds 32 Gy and supports the protocol-defined 48 Gy MTD (49).

\section{Liver}

Therapies resulting in significant liver absorbed dose include ${ }^{90} \mathrm{Y}$-microspheres (although ${ }^{90} \mathrm{Y}$-microspheres are typically treated as medical devices rather than radiopharmaceuticals under regulatory purview, they are included herein for completeness), for transarterial radioembolization, radioimmunotherapeutics using a radiometal label, somatostatin analogs, and ${ }^{131} \mathrm{I}-\mathrm{MIBG}$. Radiationinduced liver toxicity typically presents within 4-8 wk of irradiation; however, cases have been reported as early as 2 wk after irradiation and as late as 7 mo afterbirradiation (50). Classic presentation of radiation-induced liver disease (RILD) includes fatigue, abdominal pain, hepatomegaly, and ascites, in conjunction with jaundice and a rise in the level of alkaline phosphatase. These clinical symptoms are thought to be the result of hepatic "venoocclusive disease," whereby vascular congestion results in decreased oxygen delivery to the liver. Nonclassical presentations of radiation-induced liver disease are seen in patients with chronic hepatic diseases, such as cirrhosis and viral hepatitis.

MTD to liver from radioimmunotherapy exceeds $28.5 \mathrm{~Gy}$ in a single dose with ${ }^{90} \mathrm{Y}$-anti-CD20 antibodies given systemically (51). ${ }^{131}$ I-MIBG dosing has been, in part, driven by dosimetry. Mild transient hepatic function abnormalities have been seen in patients treated with MIBG. These data are complex, but doses up to $30 \mathrm{~Gy}$ to the liver have resulted in less than $10 \%$ incidence of transient reversible hepatoxicity $(52,53)$. Clinical protocols have limited dose escalation to $30 \mathrm{~Gy}$ to the liver, so liver MTD may be higher than $30 \mathrm{~Gy}$ for systemic radiopharmaceuticals. It is believed that radioantibodies and MIBG distribute uniformly through the hepatic parenchyma, resulting in a relatively uniform absorbed dose.

Being the primary target and site of accumulation, clinical use of ${ }^{90} \mathrm{Y}$-microspheres (both resin and glass) often results in whole liver mean doses more than $30-32 \mathrm{~Gy}$, which is thought to be the TD5/5 in external-beam radiotherapy. Whole liver doses of more than 42 Gy (EBRT TD50/5) are often encountered as well, although somewhat less frequently with resin ${ }^{90} \mathrm{Y}$-microspheres. Among bilobar treatments, liver toxicity modeling has indicated a $15 \%$ complication rate for mean liver doses of 35-70 Gy from glass microspheres (54). Whole liver mean doses in excess of 70 Gy from glass microspheres are known to result in $>50 \%$ chance of radiation-induced liver toxicity (54). Data from resin microspheres indicate an approximately $50 \%$ rate of toxicity for whole liver mean doses of 44-61 Gy (55). Of note is that the product insert for ${ }^{90} \mathrm{Y}$ glass microspheres (Theraspheres; Boston 
Scientific Corp.) describes delivering doses of in excess of $80 \mathrm{~Gy}$ to the targeted lobe, with recommend doses of up to $150 \mathrm{~Gy}$ to the treated lobe. Whole liver mean doses given during a single lobe or segmental infusion may not be comparable to bilobar treatments, which are more commonly seen with resin ${ }^{90} \mathrm{Y}$-microspheres. Relevant data to this point are presented in the DOSISPHERE-01 study, which compared personalized dosimetry to escalate tumor doses (mean normal liver dose of $119.7 \mathrm{~Gy}$, with 1 patient receiving $150.3 \mathrm{~Gy}$ ) with a nondosimetry group (mean liver dose of $79.2 \mathrm{~Gy}$ ) (56). Although liver function alterations occurred, they were viewed as manageable and there was only 1 case of hepatic failure in the dosimetry group $(1 / 28,3.6 \%)$. The reason why tolerable liver absorbed doses appear to be higher for microspheres in comparison to external beam or other $\beta$-emitting therapies (MIBG; radioimmunotherapies) is likely because of the microscale dosing of ${ }^{90} \mathrm{Y}$-microspheres, which tends to be nonuniformly distributed throughout the hepatic arterial system/liver. This results in many areas that receive lower absorbed doses (57). This is particularly true for ${ }^{90} \mathrm{Y}$-microsphere segmentectomies, in which $10 \%-30 \%$ of the liver often receives a mean dose in excess of 200-400 Gy. To better characterize these effects, dose-response relationships for tumors and normal liver are being refined by use of multicenter data and harmonized software/dosimetry methods among sites (58).

\section{Kidneys}

Generally, renal toxicity is defined as an increase in the serum creatinine levels, loss of creatinine clearance, or decrease in glomerular filtration rate (GFR), commonly based on the National Cancer Institute's iterations of Common Terminology Criteria for Adverse Events (CTCAE). The kidneys may be the limiting factor for the maximum cumulative activity of hydrophilic systemic RPT such as radiolabeled peptides, small molecules, or antibody fragments. The kidney tolerance of $23 \mathrm{~Gy}$, originally derived for external-beam radiotherapy, has been suggested for renal excreted radiopharmaceuticals. However, fundamental radiobiologic differences of RPTs and the derived biologic effective dose need to be considered, which may significantly vary depending on multiple factors including the half-life of the radionuclide (59). The renal dosimetry threshold has been used by studies to optimize treatment schedules by modifying the administered activity per cycle or number of cycles of ${ }^{177}$ Lu-DOTATATE $(60,61)$. These studies have demonstrated wide interpatient variability of renal absorbed dose by a factor of 10 , underscoring the importance of dosimetry. The individualized dosimetry-based methodologies have led to enhancing tumor absorbed dose with potential improvement in the patient outcome while maintaining the acceptable safety profile of the RPT.

Notably, to capture the true incidence of renal impairment after RPT a sufficient follow-up time of at minimum 6-12 mo is required (62). Therefore, the incidence and degree of renal impairment after RPT need to be considered in the context of the life expectancy of the patients. For instance, the relatively limited median overall survival of patients with advanced metastatic castrate-resistant prostate cancer (mCRPC) of 13-16 mo may not allow the occurrence of the full spectrum of renal impairment after PSMA RPT. However, the possibility of the development of renal impairment after PSMA RPT could be of more importance in earlier stages of prostate cancer $(1,63)$. Similarly, in the context of neuroendocrine neoplasms (NENs), the implication of renal toxicity may need to be considered in the context of tumor grade, especially as patients with grade 1 NENs may live a decade or longer compared with much shorter survival of patients with grade 3 NENs.

The enhanced understanding of the mechanisms of renal accumulation of radiolabeled peptides, small molecules, and antibody fragments cannot be overemphasized as this would allow devising the strategies to reduce renal toxicity. Hydrophilic radiolabeled peptides such as ${ }^{177} \mathrm{Lu}$-DOTATATE are mainly filtered by the glomeruli and partly reabsorbed by the proximal tubular cells (62). Insight into the mechanisms of renal handling of DOTA peptides led to the development of multiple strategies to reduce the renal absorbed dose (62), of which competitive inhibition of proximal tubular reabsorption by pretreatment with positively charged amino acids (arginine and lysine) has achieved a renal dose reduction of approximately $50 \%$ and is widely adopted in clinical practice $(64,65)$. In fact, with the adoption of amino acid pretreatment, the incidence of serious toxicity has been low, $\leq 1.5 \%$ grade III or IV CTCAE, regardless of the treatment schedule, the number of cycles and administered activity per cycle or cumulatively $(65,66)$.

Specific binding to PSMA of the proximal tubules appears to be the most relevant mechanism of renal retention in PSMA RLT. Using potential differential internalization rate of PSMA isoforms in the renal tubules compared with prostate cancer cells, it has been shown that small-molecule PSMA inhibitors such as 2-(phosphonomethyl) pentanedioic acid (PMPA) can displace noninternalized PSMA ligand $16 \mathrm{~h}$ after PSMA RLT in preclinical models (67). Although this appears to improve the therapeutic index of the treatment without significant reduction in the tumor absorbed dose, the translation of these findings in humans remains to be determined. Nonetheless, currently, PSMA RLT is mainly used in the advanced stage of $\mathrm{MCRPC}$ and the incidence of grade 3 or 4 renal toxicity remains low $(1 \%-3.5 \%)(1,63)$. In a phase 2 study of 28 patients who underwent ${ }^{51} \mathrm{Cr}$-EDTA GFR measurement before and 3 mo after completion of 4 cycles of ${ }^{177}$ Lu-PSMA, a very modest decline of approximately $12 \mathrm{~mL} / \mathrm{min}$ in GFR was noted $(39,68,69)$. The renal toxicity profile of PSMA RLT may be of more clinical importance in the earlier stage of prostate cancer disease continuum, higher administered activity per cycle, higher cumulative activity or use of $\alpha$-isotopes and requires further investigation. Therapeutic radioisotopes, particularly many $\alpha$-emitters, may have complicated decay schemes with daughter isotopes that can accumulate in the kidneys. This needs to be carefully considered in evaluating the therapeutic ratios of RPTs.

We believe the $23 \mathrm{~Gy}$ tolerance guidance limit from externalbeam radiation may be lower than the true tolerance of the kidneys, given the modest renal toxicity seen with modern radiopeptide therapies. We believe absorbed dose escalation studies are essential and should be strongly considered to determine whether 23 Gy represents the true limit for renal radiation-absorbed dose. Adhering to the limit derived from EBRT may result in underdosing of tumors in patients receiving RPT, compromising efficacy.

\section{Salivary Glands}

The salivary glands have long been an organ of interest related to RPT toxicity due to their being organs of accumulation and excretion of ${ }^{131}$ I, which is used to treat both hyperthyroidism and thyroid cancer. Salivary glands are highly radiosensitive, and radiation sialadenitis and xerostomia have become the most frequent complication of high-activity ${ }^{131} \mathrm{I}$ therapies for thyroid cancer, occurring in over $50 \%$ of cases (70). Typical thyroid therapy administered activities are in the range of 3.7-7.4 GBq, although cumulative activities of $>20 \mathrm{GBq}$ are sometimes used (71). Planar dosimetry has shown 
differences in radiation-absorbed doses between the parotid and submandibular glands, with median absorbed dose per administered activity of each single parotid and submandibular gland to be about $0.15 \mathrm{~Gy} / \mathrm{GBq}$ (range, $0.1-0.3 \mathrm{~Gy} / \mathrm{GBq}$ ) and $0.48 \mathrm{~Gy} / \mathrm{GBq}$ (range, $0.2-1.2 \mathrm{~Gy} / \mathrm{GBq}$ ) (72). The dosimetry of salivary glands has been investigated more intensively with the availability of ${ }^{124}$ I PET imaging, which has shown a radiation-absorbed dose of $0.23 \mathrm{~Gy} /$ GBq in patients who do not have thyroid stimulation with lemon drops (72). Absorbed dose was noted to be increased by $28 \%$ with stimulation of salivary flow. A $7.4 \mathrm{GBq}$ administration of ${ }^{131} \mathrm{I}$ would therefore deliver $1.7 \mathrm{~Gy}$, and $20 \mathrm{GBq}$ would deliver 4.6 Gy. These absorbed doses are lower than the $26 \mathrm{~Gy}$ "safe" absorbed dose to the salivary glands for external-beam radiotherapy (73). Although there is variability in methods and results for dosimetry by planar scintigraphy and PET methods, these data suggest a difference between RPT and external beam in this setting, possibly due to microdosimetry differences for ${ }^{131} \mathrm{I}$, which we do not yet fully understand (71).

The dosimetry studies of small molecules targeting PSMA such as ${ }^{124} \mathrm{I} /{ }^{131} \mathrm{I}$-MIP-1095, or ${ }^{177}$ Lu-PSMA-617 or ${ }^{177}$ Lu-PSMA I\&T have shown salivary glands commonly receive the highest radiation-absorbed dose among normal organs $(69,74)$. The underlying mechanism of tracer accumulation is likely related to the extensive expression of PSMA within the salivary glands, although there can be both specific and nonspecific binding in the salivary glands (75). Dosimetry using ${ }^{124} \mathrm{I}-\mathrm{MIP}-1095$ PET/CT to predict ${ }^{131}$ I-MIP-1095 dosimetry have shown predicted absorbed doses of 9.2-33.3 Gy from a single therapy administration (76). With ${ }^{131}$ I-MIP-1095 therapy given as a single treatment, 7 of 28 patients treated had mild and transient (3-4 wk duration) xerostomia and 1 patient had transient mucositis. In the multicenter ${ }^{177}$ Lu-PSMA-617 VISION trial, symptoms of dry mouth occurred in $38.8 \%$ of patients, but no grade 3 or greater dry mouth was observed in the 529 patients receiving a mean $37.5 \mathrm{GBq}$ over 6.9 mo (median 5 cycles of $7.4 \mathrm{GBq} /$ cycle). Dosimetry estimates for salivary gland radiation-absorbed dose from ${ }^{177}$ Lu-PSMA PET posttherapy imaging have been limited and have typically used planar imaging. Delker et al. estimated a mean of $1.4 \mathrm{~Gy} / \mathrm{GBq}$ to the salivary glands from ${ }^{177} \mathrm{Lu}-\mathrm{PSMA}-617$ therapies (77). This extrapolates to $52.5 \mathrm{~Gy}$ to the salivary glands (by extrapolation from the VISION trial), resulting in very low toxicity, but caution may be in order due to the uncertainties of planar imaging-derived dosimetry of small structures (77). Peters et al. performed ${ }^{177} \mathrm{Lu}-$ SPECT/CT dosimetry, including the salivary glands, with PSMA617 and estimated a mean absorbed dose of $0.38 \mathrm{~Gy} / \mathrm{GBq}(78)$. This dose extrapolated prescribing within the VISION trial would indicate an average salivary gland absorbed dose of $\sim 14.3 \mathrm{~Gy}$. Despite variability in the rate of xerostomia among studies, observations have typically been of low severity, usually grade 1 CTCAE (1,63). Some of the most direct evidence of the importance of radiation-absorbed dose and RBE have been in the context of $\alpha$-emitting isotope-labeled small molecules targeting PSMA. Although the antitumor effects of these agents are impressive, the incidence and severity of xerostomia appears higher and, in some instances, the main reason for toxicity-related treatment discontinuation, with over $25 \%$ of patients requesting therapy be stopped due to salivary gland toxicities (79). Of note, however, these patients had previously received ${ }^{177} \mathrm{Lu}$-PSMA targeted therapy, so the effects would need to be viewed as cumulative radiation toxicity. However, when larger molecules targeting PSMA such as antibodies are labeled to $\alpha$-isotopes, xerostomia appears to be of less concern due to low salivary gland uptake $(80)$. Various approaches have been attempted to mitigate salivary gland toxicity including sialagogues, local cooling, local injections of botulinum toxin, oral administration of monosodium glutamate, or PSMA inhibitors such as PMPA, with generally limited success and the unclear impact on tumoral uptake that require further investigation $(81,82)$. Because of the current limitations in $\alpha$-particle dosimetry, it is not possible to speculate on dose-response relationships, and these will need to be developed empirically, most likely with dosimetry obtained from diagnostic surrogates. Whole organ dose estimates from diagnostic surrogates may also then need to be informed by models of microscale $\alpha$-dosimetry.

\section{Lungs}

The most extensive studies of radiation-absorbed dose to the lungs from RPT have been undertaken in thyroid cancer. For several decades, an " $80 \mathrm{mCi}$ " rule has been in place to guide highdose ${ }^{131} \mathrm{I}$ therapies of thyroid cancer. If the whole body has $80 \mathrm{mCi}$ (2.96 GBq) or less, predicted to be present at $48 \mathrm{~h}$ after therapy, pulmonary toxicity can be avoided if patients have lung metastases from thyroid cancer. The complexity with these estimates is that when one examines the $80 \mathrm{mCi}$ rule, the predicted radiationabsorbed dose to normal lungs ranged from 57 to $112 \mathrm{~Gy}$; the photon-only portion, which better reflects the dose to normal lung parenchyma, ranged from 4.9 to 55 Gy. Thus, with ${ }^{131} \mathrm{I}$ the heterogeneity of dose means much of the $\beta$-dose substantially irradiates the tumor while the $\gamma$-dose irradiates more normal lung. In addition, the size of the lungs make a large difference (83). This area of investigation is unsettled but it does illustrate that nonuniform dose delivery can confound estimates of safe doses to organs. ${ }^{124}$ I PET imaging has been used to further inform lung radiation dosimetry (84). These data suggest that in tumor-involved lungs, higher activities might be given more safely than the activities predicted by the seminal Benua and Leeper method (85).

A dose escalation study using ${ }^{131} \mathrm{I}$-anti-CD20 antibodies and stem cell support showed that although normal lung absorbed doses under 23.75 Gy were well tolerated (other than intended myeloablative hematopoietic effects), in 2 patients who received 27.5 and 30.75 Gy to lungs - as estimated by planar imaging - significant and severe, but reversible cardiopulmonary toxicity occurred (86). These are among the few dose escalation studies reaching an MTD with RPTs.

Limited data exist regarding lung dose-toxicity relationships for ${ }^{90} \mathrm{Y}$-microsphere shunting to lungs; however, it is generally accepted that delivering over $30 \mathrm{~Gy}$ in a single treatment or over 50 Gy in sequential treatments is undesirable. These generally accepted criteria are based on very few patients, and with somewhat outdated dosimetry methods (87).

\section{Whole-Body Radiation}

A single whole-body photon exposure of 3-5 Gy produces an acute gastrointestinal syndrome and hematopoietic toxicity, which can be fatal without major medical intervention (88). Acute whole-body absorbed doses of 6-7 Gy are considered the human $\mathrm{LD}_{50 / 60}$, that is, the lethal dose for $50 \%$ of the population in $60 \mathrm{~d}$, even with supportive treatment (typically antibiotic and transfusion support) (89). Survivable whole-body doses in excess of 7-8 Gy can be reached by reducing the dose rate, or by administering the radiation in smaller fractions over several days. Before hematopoietic stem cell transplant in patients being treated for hematologic malignancies, common dose fractionation for myeloablation 
is 1.5 Gy to the whole body, twice per day, up to a total dose of 12.0-13.5 Gy (90). Whole-body dose from radiopharmaceuticals is not often an endpoint of interest due to the nonuniform nature of uptake and energy deposition; however, whole-body absorbed dose has been used effectively as a surrogate for marrow dosimetry in pediatric patients receiving ${ }^{131} \mathrm{I}-\mathrm{MIBG}$ (often 3-4 Gy over 2 treatments) (91) and in patients receiving ${ }^{131} \mathrm{I}$-tositumomab (MTD $=75$ cGy, single administration in heavily pretreated patients without major marrow involvement with tumor) $(92,93)$.

\section{SECONDARY MALIGNANCIES AND SIDE EFFECTS}

It is known that children are more susceptible to radiation effects than are adults. Indeed, the use of external-beam radiation in children has been declining over the years as a greater understanding of second malignancies and late effects on growth and development are identified along with increased efficacy of alternative cancer treatments. The use of PET imaging has allowed for elimination of external-beam radiation in many cases of Hodgkin disease, thus limiting toxicity (94) by more appropriately limiting external-beam radiation to those with residual tumor by PET. At present the main uses of RPTs in children include ${ }^{131} \mathrm{I}$ for thyroid cancer and ${ }^{131} \mathrm{I}-\mathrm{MIBG}$ for neuroblastoma. More recently, somatostatin receptor-targeting agents are being applied for RPTs of neuroblastoma. There has been use of bone-targeting agents in osteosarcoma and radiolabeled monoclonal antibodies, as well $(15,95)$.

A systematic review of the toxicities of ${ }^{131} \mathrm{I}$ therapy in patients with thyroid cancer (96) evaluated 37 articles including adults and children. Relatively early effects after treatment can include alterations in salivary and lacrimal gland function. In this review, post- ${ }^{131} \mathrm{I}$ therapy patients experienced significantly more salivary gland dysfunction (prevalence range: 16\%-54\%), lacrimal gland dysfunction (prevalence: $11 \%$ ), transient male gonadal dysfunction (prevalence: 35\%-100\%, high-level evidence), transient female gonadal dysfunction (prevalence: 28\%, low-level evidence), and second primary malignancies (prevalence: $2.7 \%-8.7 \%$, moderatelevel evidence) than unexposed patients. Breast and digestive tract cancer were the most common reported secondary malignancies. Except for the study performed by Lang et al. (97), all studies reported an increased risk of the occurrence of both solid tumors and leukemia after treatment with ${ }^{131} \mathrm{I} .{ }^{131} \mathrm{I}$ therapy seems to have no deleterious effects on female reproductive outcomes (very-low level evidence). The prevalence and severity of adverse effects were correlated to increasing cumulative ${ }^{131} \mathrm{I}$ activity. Gonadal radiation may cause transient or longer-duration azoospermia. As RPTs are used more in younger patients, sperm banking has been considered in patients receiving cumulative administrations in excess of $14 \mathrm{GBq}$ of ${ }^{131} \mathrm{I}$ (98).

In a retrospective review of the Surveillance, Epidemiology, and End Results Program (SEER) registry $(n=148,215)$, the risk for hematologic malignancies after postsurgery radioiodine treatment of well-differentiated thyroid cancer appears to be significantly higher than patients managed with surgery alone (hazard ratio, 1.43 ; $95 \% \mathrm{CI}, 1.20$ to $1.69 ; P<0.001$ ) (99); however, the absolute risk appears quite low, approximately $0.54 \%$ within $10 \mathrm{y}$ of surgery plus radioiodine therapy. In this review, no data regarding administered activities or dosimetry were available.

MIBG is increasingly a routine part of neuroblastoma therapy in the United States and some European countries. In a review of 644 neuroblastoma patients treated with ${ }^{131}$ I-MIBG (in addition to other cytotoxic therapies), the cumulative incidence of secondary malignant neoplasm (SMN) was $7.6 \%$ and $14.3 \%$ at 5 and $10 \mathrm{y}$ from first ${ }^{131} \mathrm{I}-\mathrm{MIBG}$, respectively. No increase in SMN risk was found with increased number of ${ }^{131} \mathrm{I}-\mathrm{MIBG}$ treatments or higher cumulative activity per kilogram of ${ }^{131} \mathrm{I}-\mathrm{MIBG}$ received $(P=0.72$ and $P=0.84$, respectively). Thirteen of the 19 reported SMN were hematologic. These authors concluded the cumulative risk of SMN after ${ }^{131}$ I-MIBG therapy for patients with relapsed or refractory neuroblastoma was similar to the greatest published incidence for high-risk neuroblastoma after myeloablative therapy, with no dose-dependent increase. However, there was no clear measurement of marrow absorbed dose in this study. External-beam irradiation of over 10 Gy can cause ovarian failure, which is also a toxicity that has been identified in some female patients receiving MIBG treatment (100).

A reasonably large dataset exists for prospective ${ }^{131} \mathrm{I}$-tositumomab therapy of non-Hodgkin lymphoma (101). SWOG S0016 was a phase III randomized study that compared the safety and efficacy of R-CHOP (rituximab plus cyclophosphamide, doxorubicin, vincristine, and prednisone) with CHOP-RIT (CHOP followed by consolidation with ${ }^{131}$ I-tositumomab radioimmunotherapy) for previously untreated patients with follicular lymphoma. Five hundred thirty-one previously untreated patients with follicular lymphoma were randomly assigned to receive either 6 cycles of R-CHOP or 6 cycles of CHOP-RIT. Patients in the CHOP-RIT arm had significantly better $10-\mathrm{y}$ progression-free survival than patients in the R-CHOP arm (56\% vs. $42 \% ; P=0.01)$, but $10-\mathrm{y}$ overall survival was not different between the 2 arms $(75 \%$ vs. $81 \% ; P=0.13)$. There was no significant difference between the CHOP-RIT and R-CHOP arms in regard to incidence of second malignancies $(15.1 \%$ vs. $16.1 \% ; P=0.81)$ or myelodysplastic syndrome or acute myeloid leukemia $(4.9 \%$ vs. $1.8 \% ; P=0.058)$. The estimated $10-\mathrm{y}$ cumulative incidences of death resulting from second malignancies were not different $(7.1 \%$ vs. $3.2 \% ; P=0.16)$, but cumulative incidence of death resulting from myelodysplastic syndrome or acute myeloid leukemia was higher in the CHOPRIT arm than in the R-CHOP arm (4\% vs. $0.9 \% ; P=0.02(101))$. These data support a small but measurable increased incidence of death from acute myeloid leukemia (AML) or myelodysplastic syndrome in the patients who received ${ }^{131}$ I-tostitumomab versus those who did not.

Myelodysplastic syndromes (MDS) and AML can also occur after peptide receptor radionuclide therapy for neuroendocrine tumors. Thirty cases of 1,631 patients treated over a 2 decade period were reported (102). Bodei reported a $2.35 \%$ frequency of MDS (103). Other studies have suggested a higher frequency of MDS/AML in patients who have had more extensive chemotherapy (104). It appears that the longer a patient population is observed after RPT, the greater the chance of developing MDS, possibly explaining differences among studies in the frequency of MDS/AML. Overall, the rate of MDS/acute leukemia appears to be similar to that seen from other cytotoxic systemic therapies. Further understanding of risk factors, timelines, and additive or synergistic risk from other treatments is necessary.

\section{CONFOUNDING CLINICAL FACTORS}

As RPT becomes more widely applied, there will be more clinical questions to determine how to safely combine external-beam radiation-absorbed doses with absorbed doses delivered by radiopharmaceuticals. Although this is an evolving area and thorough 
discussion is beyond the scope of the current review, concerns can include tolerances in patients who have had spinal cord or brain irradiation. Fortunately, it is rare for radiopharmaceuticals to localize substantially to the normal brain or spinal cord. However, lesions close to normal brain may present areas of risk. Similarly, there are concerns with patients who have had a large area of marrow irradiated. Such patients may have a lower marrow reserve, and increased toxicity, with external RPTs. Indeed, limitations of bone marrow irradiation have been included as eligibility criteria for Food and Drug Administration-approved radiopharmaceuticals such as ibritumomab tiuxetan, where key clinical trials excluded patients who had radiation of any type before radioimmunotherapy. Examples of efforts to combine dose planning for RPT of bone metastases with external beam radiation have been described for ${ }^{153} \mathrm{Sm}$-EDTMP as an example (105). Similar challenges will likely arise with brachytherapy, especially given the importance of this modality in prostate cancer therapy. It remains unclear how much additional absorbed dose can be given depending on the time interval after external beam radiation or RPT.

Tumor burden can also impact radiation tolerances. The clinical trials leading to the approval of anti-CD20 radioimmunotherapies intentionally excluded patients with bone marrow involvement with tumor of $>25 \%$ (92). This was arbitrary, and some trials have allowed larger amounts of tumor involvement. The admixture of tumor and normal tissue in the marrow, and elsewhere (such as the lung) can make estimations of radiation-absorbed dose to tumor and marrow challenging.

Prior and concurrent therapies can also impact radiation tolerances. Chemotherapy, especially recent, can increase the sensitivity of normal bone marrow to radiation-induced toxicities. In patients with lymphoma receiving a relatively standard bone marrow radiation-absorbed dose from radioimmunotherapy, the duration of time postchemotherapy was the most strongly correlated factor with the severity of myelosuppression (106). Although singleagent RPTs can be effective in diseases such as non-Hodgkin lymphoma, they are unlikely to provide durable disease remissions in a range of cancers. Thus, combination therapies will increasingly be tested and those studies will inform the interplay and optimal timing of RPT and other therapies including cytotoxic treatments.

As we increasingly understand DNA damage and repair, there is a growing appreciation for DNA damage from radiation. There is little specific data regarding RPT and DNA repair mechanisms. More is known regarding external-beam radiation, but at present, some of the known inherited syndromes, such as ataxia telangiectasia, ataxia-telangiectasia-like disorder, radiosensitive severe combined immunodeficiency, Nijmegen breakage syndrome, and LIG4 deficiency are associated with increased radiosensitivity $(96,107)$. This is an area where additional research is necessary.

\section{SUMMARY AND FUTURE DIRECTIONS}

The Latin phrase "Primum non nocere" means "First, do no harm." This approach is often applied to RPTs by regulators and by practitioners. It can be interpreted as "do not exceed dose limits established by external-beam radiation treatments, as harm could be done to an individual patient." However, like in chemotherapy, sometimes some reversible or addressable harm may be acceptable if there is a reasonable probability of a long-term benefit exceeding the harm. It is very interesting that in radioembolization studies of hepatic malignancies, much higher radiation-absorbed doses can be tolerated than expected from established external-beam radiation thresholds. This points to the need for dose escalation studies to better understand organ tolerance for RPTs. Underdosing patients is possible if an organ MTD is higher than expected from external-beam data. Similarly, an average dose of a therapeutic radiopharmaceutical that is safe for a population may result in systematic underdosing of a significant fraction of the population, denying individual patients an opportunity for benefit - a clear harm. We must as a field move to individualized radiopharmaceutical dosimetry-based dosing to provide better outcomes for populations who may be systematically underdosed.

\section{Gaps in Our Current Understanding}

Most currently available radiation biology data are empiric and there are extensive gaps in knowledge of the effects of radiation at the subcellular, cellular, and microenvironmental levels. The deficiencies start with limited understanding of track structure patterns of ionization and excitation resulting from various radiation types and the secondary charged particles in complex biologic media, particularly at the end of their range, where the energy transfer is most pronounced (108). It is not clear how the low and high linear energy transfer (LET) radiation affects specific subcellular targets. The most investigated is the radiation-induced damage to DNA and the repair mechanisms, including cell cycle control. However, the signals that initiate the checkpoint response, the need for cell cycle progression checkpoints for effective DNA repair, and variations in radiosensitivity throughout the cell cycle are not well characterized. Much less is known about how radiation damage to cell membranes and cellular organelles leads to radiation cytotoxicity. For instance, radiation effects on membranes may cause modifications of cell signaling pathways controlling cell response to stress, including pro- and antiapoptotic signals (109). Only recently, the effect of absorbed dose on gene expression has been investigated (110). Is remains to be studied how other radiation characteristics, such as LET and dose rate, would affect signaling pathways and gene expression. This kind of research might also provide means to identify genetic factors determining individual radiation sensitivity, which are currently unknown.

An innate characteristic of RPT is heterogeneity of dose distribution. Therefore, to understand the effect of RPT on tissues, we need to learn more about intracellular signaling and interactions between the microenvironment and bystander effects and their role in response to radiation. There is a growing interest in immunomodulatory effects of local radiotherapy on the tumor microenvironment (111). There are only limited data on the possible combination of RPT with immunotherapy. This is also the case for the combination of RPT with other therapeutic modalities, including external radiation therapy. Considering the number of unknowns listed above for any type of radiation individually, the major problem for combinations is the difficulty with assessment of the effects of combinations of different types of radiation. The same problem hinders combinations of different types of radionuclides, for example, $\alpha$-and $\beta$-emitters, to optimize RPT.

\section{KEY RECOMMENDATIONS}

The following are key recommendations:

1. Clinical adoption of dosimetry in instances in which there is considerable patient-to-patient variation in absorbed dose to organs for a given administered activity.

2. Clinical adoption of dosimetry in instances of limited organ reserve. 
3. Standardization and validation of radiopharmaceutical dosimetry approaches for organs and tumors with a goal of achieving not over $10 \%$ variability among sites — similar to what has been achieved with external-beam radiotherapy.

4. Establishment of a more nuanced balance assessment between potential benefits and toxicities (i.e., some toxicity may be necessary to achieve optimal therapeutic effect and improve patient outcome). These decisions will best be made by considering factors such as aggressiveness of underlying malignancy, life expectancy of patients, and the potential impact of toxicity on the quality of life of the patients.

5. High-quality dose escalation studies based on absorbed dose to better inform the MTD, including MTD of nonhematological organs; these data will help ensure that we do not systematically undertreat patients, thus failing to optimize antitumor effects.

6. Establishment of a registry of short- and long-term toxicities of organs related to known absorbed dose.

7. Examination of toxicities versus dose rate and radiation type (i.e., $\alpha$-emitters).

8. More thoroughly linked radiation-induced toxicities versus molecular profiles of tissues.

9. Enhanced understanding of the mechanistic normal-tissue toxicity pertinent to each RPT to devise strategies to optimize absorbed dose and rationally minimize toxicities.

10. Establishment of a systematic effort, akin to the QUANTEC or HyTEC external-beam initiatives, to better understand and catalog the dose response relationships for RPTs.

\section{DISCLOSURE}

The opinions expressed in this publication are the author(s)' own and do not reflect the view of the National Institutes of Health, the Department of Health and Human Services, or the United States government. No other potential conflict of interest relevant to this article was reported.

\section{REFERENCES}

1. Sartor O, de Bono J, Chi KN, et al. Lutetium-177-PSMA-617 for metastatic castration-resistant prostate cancer. N Engl J Med. 2021;385:1091-1103.

2. Parker C, Nilsson S, Heinrich D, et al. Alpha emitter radium-223 and survival in metastatic prostate cancer. N Engl J Med. 2013;369:213-223.

3. Bentzen SM. Theragnostic imaging for radiation oncology: dose-painting by numbers. Lancet Oncol. 2005;6:112-117.

4. Shirvani SM, Huntzinger CJ, Melcher T, et al. Biology-guided radiotherapy: redefining the role of radiotherapy in metastatic cancer. $\operatorname{Br} J$ Radiol. 2021;94: 20200873.

5. Musolino SV. Absorbed dose determination in external beam radiotherapy: an international code of practice for dosimetry based on standards of absorbed dose to water. Technical reports series no. 398. International Atomic Energy Agency (IAEA); 2000. IAEA website. https://www-pub.iaea.org/MTCD/Publications/ PDF/TRS398_scr.pdf. Accessed October 31, 2021.

6. Emami B, Lyman J, Brown A, et al. Tolerance of normal tissue to therapeutic irradiation. Int J Radiat Oncol Biol Phys. 1991;21:109-122.

7. Wessels BW, Konijnenberg MW, Dale RG, et al. MIRD pamphlet no. 20: the effect of model assumptions on kidney dosimetry and response-implications for radionuclide therapy. J Nucl Med. 2008;49:1884-1899.

8. Pouget J-P, Constanzo J. Revisiting the radiobiology of targeted alpha therapy. Front Med (Lausanne). 2021;8:692436.

9. Rich JN. Cancer stem cells in radiation resistance. Cancer Res. 2007;67:89808984.

10. Hobbs RF, Howell RW, Song H, Baechler S, Sgouros G. Redefining relative biological effectiveness in the context of the EQDX formalism: implications for alpha-particle emitter therapy. Radiat Res. 2014;181:90-98.
11. Hobbs RF, Song H, Huso DL, Sundel MH, Sgouros G. A nephron-based model of the kidneys for macro-to-micro $\alpha$-particle dosimetry. Phys Med Biol. 2012;57: 4403 .

12. Bolch WE, Eckerman KF, Sgouros G, Thomas SR. MIRD pamphlet no. 21: a generalized schema for radiopharmaceutical dosimetry-standardization of nomenclature. J Nucl Med. 2009;50:477-484.

13. Uribe C, Peterson A, Van B, et al. An international study of factors affecting variability of dosimetry calculations, part 1: design and early results of the SNMMI dosimetry challenge. J Nucl Med. 2021;62(suppl. 3):36S-47S.

14. He B, Wahl RL, Du Y, et al. Comparison of residence time estimation methods for radioimmunotherapy dosimetry and treatment planning-Monte Carlo simulation studies. IEEE Trans Med Imaging. 2008;27:521-530.

15. Plyku D, Loeb DM, Prideaux AR, et al. Strengths and weaknesses of a planar whole-body method of ${ }^{153} \mathrm{Sm}$ dosimetry for patients with metastatic osteosarcoma and comparison with three-dimensional dosimetry. Cancer Biother Radiopharm. 2015;30:369-379.

16. Wahl RL. The clinical importance of dosimetry in radioimmunotherapy with tositumomab and iodine I 131 tositumomab. Semin Oncol. 2003;30(suppl 4):31-38.

17. Kam WW-Y, Banati RB. Effects of ionizing radiation on mitochondria. Free Radic Biol Med. 2013;65:607-619.

18. Jonathan EC, Bernhard EJ, McKenna WG. How does radiation kill cells? Curr Opin Chem Biol. 1999;3:77-83.

19. Sia J, Szmyd R, Hau E, Gee HE. Molecular mechanisms of radiation-induced cancer cell death: a primer. Front Cell Dev Biol. 2020;8:41.

20. McBride WH, Schaue D. Radiation-induced tissue damage and response. J Pathol. 2020;250:647-655.

21. Sgouros G, Roeske JC, McDevitt MR, et al. MIRD pamphlet no. 22 (abridged): radiobiology and dosimetry of alpha-particle emitters for targeted radionuclide therapy. J Nucl Med. 2010;51:311-328.

22. Pugliese MD, Grossi GF, Monforti F, et al. Inactivation of individual mammalian cells by single alpha-particles. Int J Radiat Biol. 1997;72:397-407.

23. Sgouros G. Dosimetry, radiobiology and synthetic lethality: radiopharmaceutical therapy (RPT) with alpha-particle-emitters. Semin Nucl Med. 2020;50:124-132.

24. Cremonesi M, Botta F, Di Dia A, et al. Dosimetry for treatment with radiolabelled somatostatin analogues: a review. Q J Nucl Med Mol Imaging. 2010;54:37.

25. Marks LB, Yorke ED, Jackson A, et al. Use of normal tissue complication probability models in the clinic. Int J Radiat Oncol Biol Phys. 2010;76:S10-S19.

26. Grimm J, Marks LB, Jackson A, Kavanagh BD, Xue J, Yorke E. High Dose per Fraction, Hypofractionated Treatment Effects in the Clinic (HyTEC): an overview. Int J Radiat Oncol Biol Phys. 2021;110:1-10.

27. Rubin P, Casarett G. Direction for clinical radiation pathology: the tolerance dose. Front Radiat Ther Oncol. 1972;6:1-16.

28. Capala J, Graves S, Scott A, et al. Dosimetry for radiopharmaceutical therapy: current practices and commercial resources. J Nucl Med. 2021;62(suppl 3): $3 \mathrm{~S}-11 \mathrm{~S}$.

29. Sgouros G, Dewaraja YK, Escorcia F, et al. Tumor response to radiopharmaceutical therapies: the knowns and the unknowns. J Nucl Med. 2021;62(suppl 3):12S$22 \mathrm{~S}$.

30. Sgouros G, Stabin M, Erdi Y, et al. Red marrow dosimetry for radiolabeled antibodies that bind to marrow, bone, or blood components. Med Phys. 2000;27: $2150-2164$

31. Hindorf C, Glatting G, Chiesa C, Linden O, Flux G. EANM Dosimetry Committee guidelines for bone marrow and whole-body dosimetry. Eur J Nucl Med Mol Imaging. 2010;37:1238-1250.

32. Sgouros G, Jureidini IM, Scott AM, Graham MC, Larson SM, Scheinberg DA. Bone marrow dosimetry: regional variability of marrow-localizing antibody. J Nucl Med. 1996;37:695-698.

33. Schwartz J, Humm JL, Divgi CR, Larson SM, O’Donoghue JA. Bone marrow dosimetry using ${ }^{124}$ I-PET. J Nucl Med. 2012;53:615-621.

34. Hindorf C, Lind Ã. Con O, Tennvall J, WingÃ ¥¥rdh K, Strand S-E. Evaluation of methods for red marrow dosimetry based on patients undergoing radioimmunotherapy. Acta Oncol. 2005;44:579-588.

35. Sgouros G. Bone marrow dosimetry for radioimmunotherapy: theoretical considerations. J Nucl Med. 1993;34:689-694.

36. O'Donoghue JA, Baidoo N, Deland D, Welt S, Divgi CR, Sgouros G. Hematologic toxicity in radioimmunotherapy: dose-response relationships for I-131 labeled antibody therapy. Cancer Biother Radiopharm. 2002;17:435443.

37. Hindorf C, Linden O, Tennvall J, Wingardh K, Strand SE. Time dependence of the activity concentration ratio of red marrow to blood and implications for red marrow dosimetry. Cancer. 2002;94(4, Suppl)1235-1239.

38. Cremonesi M, Ferrari M, Di Dia A, et al. Recent issues on dosimetry and radiobiology for peptide receptor radionuclide therapy. $Q \mathrm{~J} \mathrm{Nucl} \mathrm{Med} \mathrm{Mol} \mathrm{Imaging.}$ 2011;55:155-167. 
39. Violet J, Jackson P, Ferdinandus J, et al. Dosimetry of Lu-177-PSMA-617 in metastatic castration-resistant prostate cancer: correlations between pretherapeutic imaging and whole-body tumor dosimetry with treatment outcomes. $J$ Nucl Med. 2019;60:517-523.

40. Lawal I, Vorster M, Boshomane T, Ololade K, Ebenhan T, Sathekge M. Metastatic prostate carcinoma presenting as a superscan on Ga-68-PSMA PET/CT. Clin Nucl Med. 2015;40:755-756.

41. Sgouros G, Graham MC, Divgi CR, Larson SM, Scheinberg DA. Modeling and dosimetry of monoclonal antibody M195 (anti-CD33) in acute myelogenous leukemia. J Nucl Med. 1993;34:422-430.

42. Glatting G, Bardies M, Lassmann M. Treatment planning in molecular radiotherapy. Z Med Phys. 2013;23:262-269.

43. Frey E, He B, Sgouros G, Flinn I, Wahl R. Estimation of post-therapy marrow dose rate in myeloablative Y-90 ibritumomab tiuxetan therapy. J Nucl Med. 2006; 47(suppl 1):156P.

44. Wahl RL, Zasadny KR, MacFarlane D, et al. Iodine-131 anti-B1 antibody for B-cell lymphoma: an update on the Michigan Phase I experience. J Nucl Med. 1998;39(8, Suppl)21S-27S.

45. Hobbs RF, Song H, Watchman CJ, et al. A bone marrow toxicity model for ${ }^{223} \mathrm{Ra}$ alpha-emitter radiopharmaceutical therapy. Phys Med Biol. 2012;57:3207-3222.

46. Grant D, Bjerke R, Wittke K, et al. Pharmacokinetics and dosimetry of BAY 1862864, an alpha-emitting targeted thorium conjugate (CD22-TTC) in the Cynomolgus monkey. Eur J Nucl Med Mol Imaging. 2018;45:S124.

47. Sgouros G, Allen BJ, Back T, et al. MIRD Monograph: Radiobiology and Dosimetry for Radiopahrmaceutical Therapy with Alpha-Particle Emitters. Sgouros G, ed. Reston VA: Society of Nuclear Medicine and Molecular Imaging; 2015.

48. Kaminski MS, Radford JA, Gregory SA, et al. Re-treatment with I-131 tositumomab in patients with non-Hodgkin's lymphoma who had previously responded to I-131 tositumomab. J Clin Oncol. 2005;23:7985-7993.

49. Passalaqua S, Natwa M, Chen M-K, et al. Relationship of marrow radiation dose and timing of engraftment for targeted radioimmunotherapy with anti-CD45 iodine $\left({ }^{131} \mathrm{I}\right)$ apamistamab [Iomab-B] in patients with active relapsed or refractory acute myeloid leukemia. $J$ Nucl Med. 2021;62(suppl 1):82.

50. Kim J, Jung Y. Radiation-induced liver disease: current understanding and future perspectives. Exp Mol Med. 2017;49:e359.

51. Wahl RL, Frey EC, Jacene HA, et al. Prospective SPECT-CT organ dosimetrydriven radiation-absorbed dose escalation using the in-111 (111in)/yttrium 90 $(90 y)$ ibritumomab tiuxetan (Zevalin $囚)$ theranostic pair in patients with lymphoma at myeloablative dose levels. Cancers (Basel). 2021;13:2828.

52. Quach A, Ji L, Mishra V, et al. Thyroid and hepatic function after high-dose 131I-metaiodobenzylguanidine (131I-MIBG) therapy for neuroblastoma. Pediatr Blood Cancer. 2011;56:191-201.

53. Matthay KK, Weiss B, Villablanca JG, et al. Dose escalation study of no-carrieradded 131I-metaiodobenzylguanidine for relapsed or refractory neuroblastoma: new approaches to neuroblastoma therapy consortium trial. J Nucl Med. 2012;53: 1155-1163.

54. Chiesa C, Mira M, Maccauro M, et al. A dosimetric treatment planning strategy in radioembolization of hepatocarcinoma with $90 \mathrm{Y}$ glass microspheres. $Q \mathrm{~J} \mathrm{Nucl}$ Med Mol Imaging. 2012;56:503-508.

55. Strigari L, Sciuto R, Rea S, et al. Efficacy and toxicity related to treatment of hepatocellular carcinoma with 90Y-SIR spheres: radiobiologic considerations. J Nucl Med. 2010;51:1377-1385.

56. Garin E, Tzelikas L, Guiu B, et al. Major impact of personalized dosimetry using ${ }^{90} \mathrm{Y}$ loaded glass microspheres SIRT in HCC: Final overall survival analysis of a multicenter randomized phase II study (DOSISPHERE-01). J Clin Oncol. 2020; $8(4$, suppl):516.

57. Walrand S, Hesse M, Chiesa C, Lhommel R, Jamar F. The low hepatic toxicity per Gray of ${ }^{90} \mathrm{Y}$ glass microspheres is linked to their transport in the arterial tree favoring a nonuniform trapping as observed in posttherapy PET imaging. $J$ Nucl Med. 2014;55:135-140.

58. Lam M, Salem R, Garin E. Abstract No. LB02 A global study of advanced dosimetry in the treatment of hepatocellular carcinoma with Yttrium-90 glass microspheres: analyses from the TARGET study. J Vasc Interv Radiol. 2021;32:S42.

59. Oehme L, Dorr W, Wust P, Kotzerke J. [Influence of time-dose-relationships in therapeutic nuclear medicine applications on biological effectiveness of irradiation: consequences for dosimetry]. Nucl Med (Stuttg). 2008;47:205-209.

60. Garske-Román U, Sandström M, Fröss Baron K, et al. Prospective observational study of (177)Lu-DOTA-octreotate therapy in 200 patients with advanced metastasized neuroendocrine tumours (NETs): feasibility and impact of a dosimetryguided study protocol on outcome and toxicity. Eur J Nucl Med Mol Imaging. 2018;45:970-988.

61. Del Prete M, Buteau F-A, Arsenault F, et al. Personalized ${ }^{177}$ Lu-octreotate peptide receptor radionuclide therapy of neuroendocrine tumours: initial results from the P-PRRT trial. Eur J Nucl Med Mol Imaging. 2019;46:728-742.
62. Vegt E, de Jong M, Wetzels JF, et al. Renal toxicity of radiolabeled peptides and antibody fragments: mechanisms, impact on radionuclide therapy, and strategies for prevention. J Nucl Med. 2010;51:1049-1058.

63. Hofman MS, Emmett L, Sandhu S, et al. [ $\left.{ }^{177} \mathrm{Lu}\right]$ Lu-PSMA-617 versus cabazitaxel in patients with metastatic castration-resistant prostate cancer (TheraP): a randomised, open-label, phase 2 trial. Lancet. 2021;397:797-804.

64. Rolleman EJ, Valkema R, de Jong M, Kooij PP, Krenning EP. Safe and effective inhibition of renal uptake of radiolabelled octreotide by a combination of lysine and arginine. Eur J Nucl Med Mol Imaging. 2003;30:9-15.

65. Strosberg J, El-Haddad G, Wolin E, et al. Phase 3 Trial of (177)Lu-Dotatate for Midgut Neuroendocrine Tumors. N Engl J Med. 2017;376:125-135.

66. Kwekkeboom DJ, Teunissen JJ, Bakker WH, et al. Radiolabeled somatostatin analog $\left[{ }^{177} \mathrm{Lu}\right.$-DOTA0,Tyr3]octreotate in patients with endocrine gastroenteropancreatic tumors. J Clin Oncol. 2005;23:2754-2762.

67. Kratochwil C, Giesel FL, Leotta K, et al. PMPA for nephroprotection in PSMAtargeted radionuclide therapy of prostate cancer. J Nucl Med. 2015;56:293-298.

68. Violet J, Sandhu S, Iravani A, et al. Long term follow-up and outcomes of re-treatment in an expanded 50 patient single-center phase II prospective trial of ${ }^{177}$ Lu-PSMA-617 theranostics in metastatic castrate-resistant prostate cancer. J Nucl Med. 2020;61:857-865.

69. Violet J, Jackson P, Ferdinandus J, et al. Dosimetry of (177)Lu-PSMA-617 in metastatic castration-resistant prostate cancer: correlations between pretherapeutic imaging and whole-body tumor dosimetry with treatment outcomes. $J \mathrm{NuCl} \mathrm{Med}$. 2019;60:517-523.

70. Nakada K, Ishibashi T, Takei T, et al. Does lemon candy decrease salivary gland damage after radioiodine therapy for thyroid cancer? J Nucl Med. 2005;46:261-266.

71. Jentzen W, Schneider E, Freudenberg L, et al. Relationship between cumulative radiation dose and salivary gland uptake associated with radioiodine therapy of thyroid cancer. Nucl Med Commun. 2006;27:669-676.

72. Jentzen W, Balschuweit D, Schmitz J, et al. The influence of saliva flow stimulation on the absorbed radiation dose to the salivary glands during radioiodine therapy of thyroid cancer using 124 I PET (/CT) imaging. Eur J Nucl Med Mol Imaging. 2010;37:2298-2306.

73. Eisbruch A, Ten Haken RK, Kim HM, Marsh LH, Ship JA. Dose, volume, and function relationships in parotid salivary glands following conformal and intensity-modulated irradiation of head and neck cancer. Int J Radiat Oncol Biol Phys. 1999;45:577-587.

74. Okamoto S, Thieme A, Allmann J, et al. Radiation dosimetry for (177)Lu-PSMA I\&T in metastatic castration-resistant prostate cancer: absorbed dose in normal organs and tumor lesions. J Nucl Med. 2017;58:445-450.

75. Rupp NJ, Umbricht CA, Pizzuto DA, et al. First clinicopathologic evidence of a non-psma-related uptake mechanism for (68)Ga-PSMA-11 in salivary glands. J Nucl Med. 2019;60:1270-1276.

76. Zechmann CM, Afshar-Oromieh A, Armor T, et al. Radiation dosimetry and first therapy results with a 124 I/131 I-labeled small molecule (MIP-1095) targeting PSMA for prostate cancer therapy. Eur J Nucl Med Mol Imaging. 2014;41: 1280-1292.

77. Delker A, Fendler WP, Kratochwil C, et al. Dosimetry for 177 Lu-DKFZ-PSMA617: a new radiopharmaceutical for the treatment of metastatic prostate cancer. Eur J Nucl Med Mol Imaging. 2016;43:42-51.

78. Peters SM, Privé BM, de Bakker M, et al. Intra-therapeutic dosimetry of $\left[{ }^{177} \mathrm{Lu}\right]$ Lu-PSMA-617 in low-volume hormone-sensitive metastatic prostate cancer patients and correlation with treatment outcome. Eur J Nucl Med Mol Imaging. July 4, 2021 [Epub ahead of print].

79. Feuerecker B, Tauber R, Knorr K, et al. Activity and adverse events of actinium225-PSMA-617 in advanced metastatic castration-resistant prostate cancer after failure of lutetium-177-PSMA. Eur Urol. 2021;79:343-350.

80. Tagawa ST, Osborne J, Fernandez E, et al. Phase I dose-escalation study of PSMA-targeted alpha emitter ${ }^{225}$ Ac-J591 in men with metastatic castrationresistant prostate cancer (mCRPC). J Clin Oncol. 2020;38(15, suppl);5560

81. Iravani A, Violet J, Azad A, Hofman MS. Lutetium-177 prostate-specific membrane antigen (PSMA) theranostics: practical nuances and intricacies. Prostate Cancer Prostatic Dis. 2020;23:38-52.

82. Harsini S, Saprunoff H, Alden T, Mohammadi B, Wilson D, Benard F. The effects of monosodium glutamate on PSMA radiotracer uptake in men with recurrent prostate cancer: a prospective, randomized, double-blind, placebo-controlled intraindividual imaging study. J Nucl Med. 2021;62:81-87.

83. Sgouros G, Song H, Ladenson PW, Wahl RL. Lung toxicity in radioiodine therapy of thyroid carcinoma: development of a dose-rate method and dosimetric implications of the 80-mCi rule. J Nucl Med. 2006;47:1977-1984.

84. Hobbs RF, Wahl RL, Lodge MA, et al. ${ }^{124}$ I PET-based 3D-RD dosimetry for a pediatric thyroid cancer patient: real-time treatment planning and methodologic comparison. J Nucl Med. 2009;50:1844-1847. 
85. Benua RS, Cicale NR, Sonenberg M, Rawson R. The relation of radioiodine dosimetry to results and complications in the treatment of metastatic thyroid cancer. Am J Roentgenol Radium Ther Nucl Med. 1962;87:171-182.

86. Press OW, Eary JF, Appelbaum FR, et al. Radiolabeled-antibody therapy of B-cell lymphoma with autologous bone marrow support. $N$ Engl J Med. 1993; 329:1219-1224.

87. Ho S, Lau W, Leung T, Chan M, Johnson P, Li A. Clinical evaluation of the partition model for estimating radiation doses from yttrium-90 microspheres in the treatment of hepatic cancer. Eur J Nucl Med. 1997;24:293-298.

88. Waselenko JK, MacVittie TJ, Blakely WF, et al. Medical management of the acute radiation syndrome: recommendations of the Strategic National Stockpile Radiation Working Group. Ann Intern Med. 2004;140:1037-1051.

89. Anno GH, Young R, Bloom R, Mercier J. Dose response relationships for acute ionizing-radiation lethality. Health Phys. 2003;84:565-575.

90. Wong JY, Filippi AR, Dabaja BS, Yahalom J, Specht L. Total body irradiation: guidelines from the international lymphoma radiation oncology group (ILROG). Int J Radiat Oncol Biol Phy. 2018;101:521-529.

91. Pandit-Taskar N, Zanzonico P, Hilden P, Ostrovnaya I, Carrasquillo JA, Modak S. Assessment of organ dosimetry for planning repeat treatments of high-dose ${ }^{131}$ I-MIBG therapy: ${ }^{123}$ I-MIBG vs. post-therapy ${ }^{131}$ I-MIBG imaging. Clin Nucl Med. 2017;42:741.

92. Kaminski MS, Zasadny KR, Francis IR, et al. Iodine-131-anti-B1 radioimmunotherapy for B-cell lymphoma. J Clin Oncol. 1996;14:1974-1981.

93. Kaminski MS, Estes J, Zasadny KR, et al. Radioimmunotherapy with iodine 131I tositumomab for relapsed or refractory B-cell non-Hodgkin lymphoma: updated results and long-term follow-up of the University of Michigan experience. Blood. 2000;96:1259-1266.

94. Jairam V, Roberts KB, James BY. Historical trends in the use of radiation therapy for pediatric cancers: 1973-2008. Int J Radiat Oncol Biol Phys. 2013;85(3): e151-e155.

95. Jacene H, Crandall J, Kasamon YL, et al. Initial experience with tositumomab and I-131-labeled tositumomab for treatment of relapsed/refractory Hodgkin lymphoma. Mol Imaging Biol. 2017;19:429-436.

96. Clement SC, Peeters R, Ronckers C, et al. Intermediate and long-term adverse effects of radioiodine therapy for differentiated thyroid carcinoma-a systematic review. Cancer Treat Rev. 2015;41:925-934.

97. Lang BH, Wong IO, Wong KP, Cowling BJ, Wan KY. Risk of second primary malignancy in differentiated thyroid carcinoma treated with radioactive iodine therapy. Surgery. 2012;151:844-850.

98. Krassas GE, Pontikides N. Gonadal effect of radiation from ${ }^{131} \mathrm{I}$ in male patients with thyroid carcinoma. Arch Androl. 2005;51:171-175.

99. Molenaar RJ, Sidana S, Radivoyevitch T, et al. Risk of hematologic malignancies after radioiodine treatment of well-differentiated thyroid cancer. $J$ Clin Oncol. 2018;36:1831-1839.

100. Clement SC, Kraal K, van Eck-Smit B, et al. Primary ovarian insufficiency in children after treatment with ${ }^{131} \mathrm{I}$-metaiodobenzylguanidine for neuroblastoma: report of the first two cases. J Clin Endocrinol Metab. 2014;99:E112-E116.

101. Shadman M, Li H, Rimsza L, et al. Continued excellent outcomes in previously untreated patients with follicular lymphoma after treatment with CHOP plus rituximab or CHOP plus ${ }^{131}$ I-tositumomab: long-term follow-up of phase III randomized study SWOG-S0016. J Clin Oncol. 2018;36:697.

102. Chantadisai M, Kulkarni H, Baum R. Therapy-related myeloid neoplasm after peptide receptor radionuclide therapy (PRRT) in 1631 patients from our 20 years of experiences: Prognostic parameters and overall survival. Eur J Nucl Med Mol Imaging. 2021;48:1390-1398.

103. Bodei L, Kidd M, Paganelli G, et al. Long-term tolerability of PRRT in 807 patients with neuroendocrine tumours: the value and limitations of clinical factors. Eur J Nucl Med Mol Imaging. 2015;42:5-19.

104. Brieau B, Hentic O, Lebtahi R, et al. High risk of myelodysplastic syndrome and acute myeloid leukemia after ${ }^{177}$ Lu-octreotate PRRT in NET patients heavily pretreated with alkylating chemotherapy. Endocr Relat Cancer. 2016; 23:L17-L23.

105. Hobbs RF, McNutt T, Baechler S, et al. A treatment planning method for sequentially combining radiopharmaceutical therapy and external radiation therapy. Int J Radiat Oncol Biol Phys. 2011;80:1256-1262.

106. Baechler S, Hobbs RF, Jacene HA, Bochud FO, Wahl RL, Sgouros G. Predicting hematologic toxicity in patients undergoing radioimmunotherapy with ${ }^{90}$ Y-ibritumomab tiuxetan or ${ }^{131}$ I-tositumomab. J Nucl Med. 2010;51:1878-1884.

107. Seibold P, Auvinen A, Averbeck D, et al. Clinical and epidemiological observations on individual radiation sensitivity and susceptibility. Int J Radiat Biol. 2020;96:324-339.

108. Ahmed MM, Coleman CN, Mendonca M, et al. Workshop report for cancer research: Defining the shades of Gy: Utilizing the biological consequences of radiotherapy in the development of new treatment approaches - meeting viewpoint. Cancer Res. 2018;78:2166-2170.

109. Eichholtz-Wirth H, Sagan D. Altered signaling of TNFalpha-TNFR1 and SODD/BAG4 is responsible for radioresistance in human HT-R15 cells. Anticancer Res. 2002;22(1A):235-240.

110. John-Aryankalayil M, Palayoor ST, Makinde AY, et al. Fractionated radiation alters oncomir and tumor suppressor miRNAs in human prostate cancer cells. Radiat Res. 2012;178:105-117.

111. Buchwald ZS, Wynne J, Nasti TH, et al. Radiation, immune checkpoint blockade and the abscopal effect: a critical review on timing, dose and fractionation. Front Oncol. 2018;8:612.

112. Sangro B, Gil-Alzugaray B, Rodriguez J, et al. Liver disease induced by radioembolization of liver tumors: description and possible risk factors. Cancer. 2008;112:1538-1546.

113. Trip AK, Sikorska K, van Sandick JW, et al. Radiation-induced dose-dependent changes of the spleen following postoperative chemoradiotherapy for gastric cancer. Radiother Oncol. 2015;116:239-244.

114. Verburg FA, Stokkel MP, Düren C, et al. No survival difference after successful 131 I ablation between patients with initially low-risk and high-risk differentiated thyroid cancer. Eur J Nucl Med Mol Imaging. 2010;37:276-283.

115. Hartung-Knemeyer V, Nagarajah J, Jentzen W, et al. Pre-therapeutic blood dosimetry in patients with differentiated thyroid carcinoma using 124-iodine: predicted blood doses correlate with changes in blood cell counts after radioiodine therapy and depend on modes of TSH stimulation and number of preceding radioiodine therapies. Ann Nucl Med. 2012;26:723-729. 